\title{
Coleções biológicas: Levantamento de conhecimentos prévios de acadêmicos de Ciências biológicas do Instituto Federal de Rondônia e Facultad de Humanidades y Ciencias de la Educación de La Plata.
}

David Dietrich Neto (IFRO) - davidietrich52@ gmail.com Marco Rodrigo de Souza (IFRO) - marco.souza@ifro.edu.br

Resumo: A pesquisa foi realizada na Facultad de Humanidades y Ciencias de la Educación de La Plata e no Instituto Federal de Educação, Ciência e Tecnologia de Rondônia- Campus Colorado do Oeste com os acadêmicos de profesorado/licenciatura em Ciências Biológicas. O trabalho foi feito através de pesquisa qualitativa. Neste enfoque foi realizada uma pesquisa através de questionário, a fim de realizar um levantamento para avaliar os conhecimentos prévios dos acadêmicos participantes, com a finalidade de comparar o nivel de conhecimentos, com enfoque nas coleções biológicas. De acordo com os resultados é possivel notar que tanto na Universidade de La Plata quanto no Instituto Federal de Rondônia houve uma porcentagem alta de acadêmicos que deixaram nulas as perguntas de conhecimentos sobre Coleções Biológicas e Coleções Biológicas virtuais, mas responderam que utilizaram em certas disciplinas, diante disso conclui-se que pode ter ocorrido uma falha na interpretação dos questionários e/ou defasagem na parte teórica do conteúdo.

Palavras-chave: Coleções Biológicas. Formação acadêmica. Conhecimentos prévios.

Resumem: La investigación fue realizada en la Facultad de Humanidades y Ciencias de la Educación de La Plata y el Instituto Federal de Educação, Ciência e Tecnologia de Rondônia Campus Colorado do Oeste con estudiantes de profesorado/licenciatura en ciencias biológicas. El trabajo se realizó a través de la investigación cualitativa. En este enfoque, se realizó una investigación a través de un cuestionario, con el fin de realizar un levantamiento para evaluar el conocimiento previo de los académicos participantes, con el fin de comparar el nivel de conocimiento, centrándose en colecciones biológicas. Según los resultados, es posible notar que tanto los académicos de la Universidad de La Plata como del Instituto Federal de Rondônia tenían un alto porcentaje de académicos que dejaban nulas las preguntas sobre el conocimiento sobre las Colecciones biológicas y las Colecciones biológicas virtuales, pero respondieron que las utilizaban en ciertos disciplinas, se concluye que puede haber habido una mala interpretación de los cuestionarios y / o desafío en la parte teórica del contenido. 
Palabras clave: Colecciones Biológicas. Formación académica. Conocimientos previos.

\section{Introdução}

As coleções biológicas são constituídas por organismos vivos ou mortos, preservados para estudos taxonômicos, sendo preservado o seu material genético. Contribuindo no conhecimento e na conservação da biodiversidade. (GARCIA et. al). De acordo com (ARANDA) A coleção biológica pode ser entendida como um conjunto de organismos que são preservados fora do ambiental natural, ou seja, o seu local de coleta, sendo esses organismos organizados para a identificação taxonômica de cada um dos espécimes. Além do conhecimento cientifico através da identificação, as coleções biológicas também desempenham papel relevante para a saúde pública e para outros setores econômicos, a partir dos dados biológicos é possível prever o aparecimento de pragas agrícolas, doenças humanas, o que possibilita uma maior eficácia no combate contra as epidemias. (PEIXOTO, 2006)

Através das coleções biológicas é possível que haja uma sensibilização por parte dos alunos, sendo uma forma de promover a conscientização significativa para a Educação Ambiental, tornando o ensino mais atrativo, levando-os a participarem na prática da conservação das espécimes, dessa forma os alunos tem uma melhor compreensão por ter um contato direto com o material estudado, após ser realizado a coleta dos espécimes e confeccionado a coleção biológica. (WOMMER, 2013) Todo o material biológico coletado segue um fluxo de triagem, identificação, depósito, ou tombamento, o que permite sua disponibilização para a comunidade científica, (ARANDA,2014).

De acordo (ZAHER et. Al) É nas coleções científicas que encontramos representantes da fauna já extinta, que habitou um dia os ecossistemas alterados de forma irreversível pela ação antrópica. As coleções constituem uma base de dados essencial para os estudos de caracterização e impacto ambiental.

\footnotetext{
As coleções a serem apoiadas são aquelas que têm como missão institucional explícita a formação, conservação e o crescimento de seus acervos biológicos e que atendem, em base rotineira, à demanda de permuta de exemplares, ao intercâmbio de identificações e às solicitações dos especialistas (PEIXOTO, 2006)
}

Uma das melhores formas de se aprender sobre a biodiversidade é através das coleções biológicos, assim como (TRIPLEHORN e JOHNSON, 2011) diz sobre a melhor forma de aprender sobre os insetos, o manuseio dos espécimes e a preparação de coleções revelam muitas coisas que não podem ser ensinadas nos livros de texto. Muitas pessoas 
acreditam que colecionar insetos é extremamente interessante porque fornece não apenas a satisfação de estar em campo, mas também de aprender na prática.

Este trabalho teve início durante um intercambio realizado na Universidade de La Plata na Argentina, onde foi aplicado um questionário para os acadêmicos sobre as coleções biológicas virtuais, o questionário serviu para identificar os conhecimentos sobre o assunto que os acadêmicos possuíam e com intuito de saber se as coleções biológicas faziam parte da vida estudantil. Sendo assim, este trabalho teve por objetivo analisar os conhecimentos prévios das turmas de Licenciatura em Ciências Biológicas do Instituto Federal de Rondônia, com a finalidade de comparar com o nível de conhecimentos dos acadêmicos da Universidade de La Plata.

\section{Material e métodos}

Esse trabalho é de cunho qualitativo, pois objetiva aprofundar-se na compreensão dos fenômenos que estuda, interpretando-os segundo a perspectiva dos próprios sujeitos que participam da situação, sem se preocupar com representatividade numérica, generalizações estatísticas e relações lineares de causa e efeito (GUERRA, 2014).

A pesquisa foi realizada no Instituto Federal de Educação, Ciência e Tecnologia de Rondônia - Campus Colorado do Oeste, com os acadêmicos de Ciências Biológicas, sendo também aplicado durante a mobilidade para os acadêmicos da Universidade de La Plata, Argentina.

Diante da permissão dos professores presentes durante a aula e termo de consentimento de participação da pesquisa devidamente assinado pelos discentes participantes, foi aplicado um questionário contendo questões de múltiplas escolhas e dissertativas, abordando sobre os conhecimentos e métodos de utilização das coleções biológicas que os discentes já haviam presenciado.

Logo após foi realizada a tabulação dos dados, em porcentagem, com auxílio do programa Microsoft Office Excel e posteriormente o registro desses dados nesse artigo científico.

\section{Resultados e discussões}

Um grupo de centro e três acadêmicos participou da pesquisa, sendo setenta e um do Brasil e trinta e dois da Argentina. Através do questionário aplicado foi possível mensurar os 
conhecimentos prévios de ambas as turmas sobre Coleções Biológicas. Sendo então possível realizar uma comparação entre os acadêmicos de Ciências Biológicas de ambos os países.

Questão 1 - Vocês conhecem Coleções Biológicas?

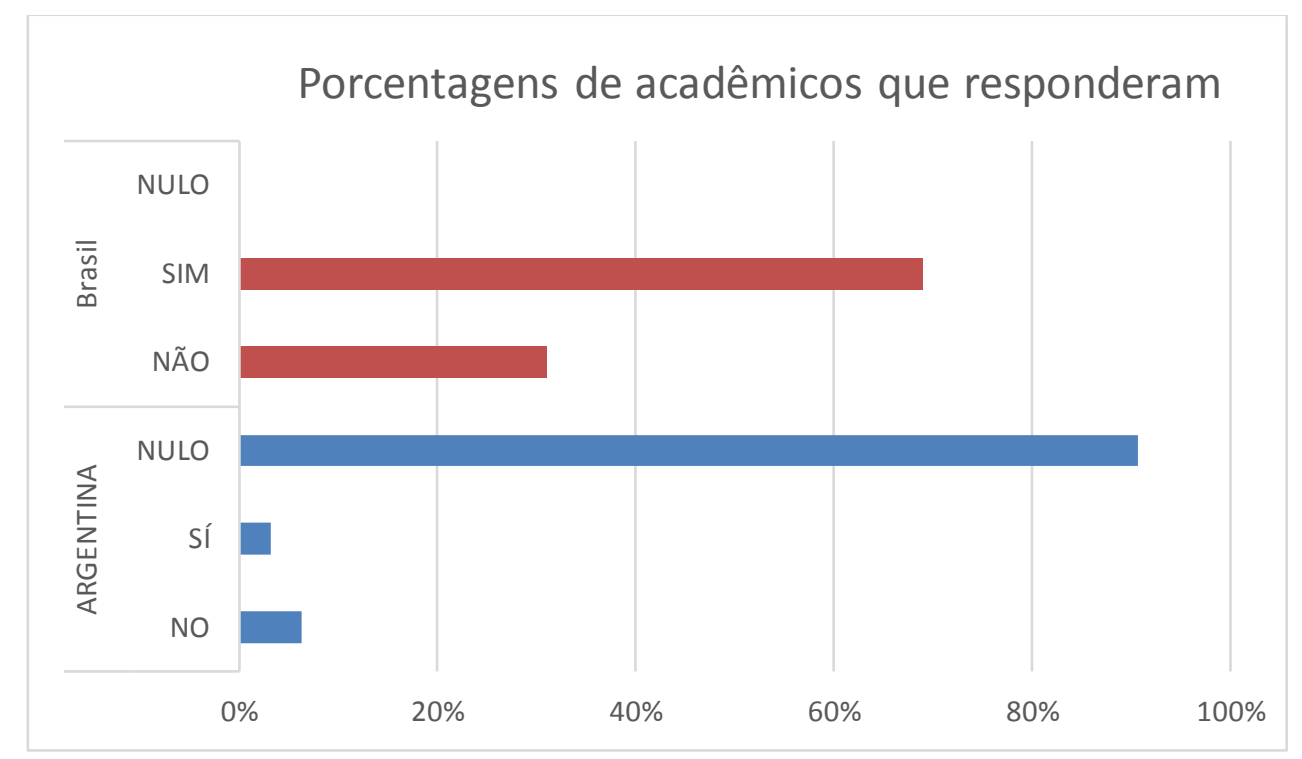

Figura 1: Respostas dos acadêmicos.

Na primeira questão foi questionado aos alunos se eles conheciam Coleções Biológicas, os acadêmicos brasileiros responderam $69 \%$ que sim, $31 \%$ que não, sendo que os acadêmicos argentinos responderam $91 \%$ nulo, $3 \%$ que sim e $6 \%$ que não.

De acordo com WOMMER (2013) As coleções biológicas promovem o conhecimento e o reconhecimento de conteúdo do dia a dia da disciplina de Ciências visando à preservação e conservação da biodiversidade presente e trazendo para dentro da sala de aula a realidade da comunidade onde a escola está inserida.

Questão 2 - Você conhece coleções biológicas virtuais? 


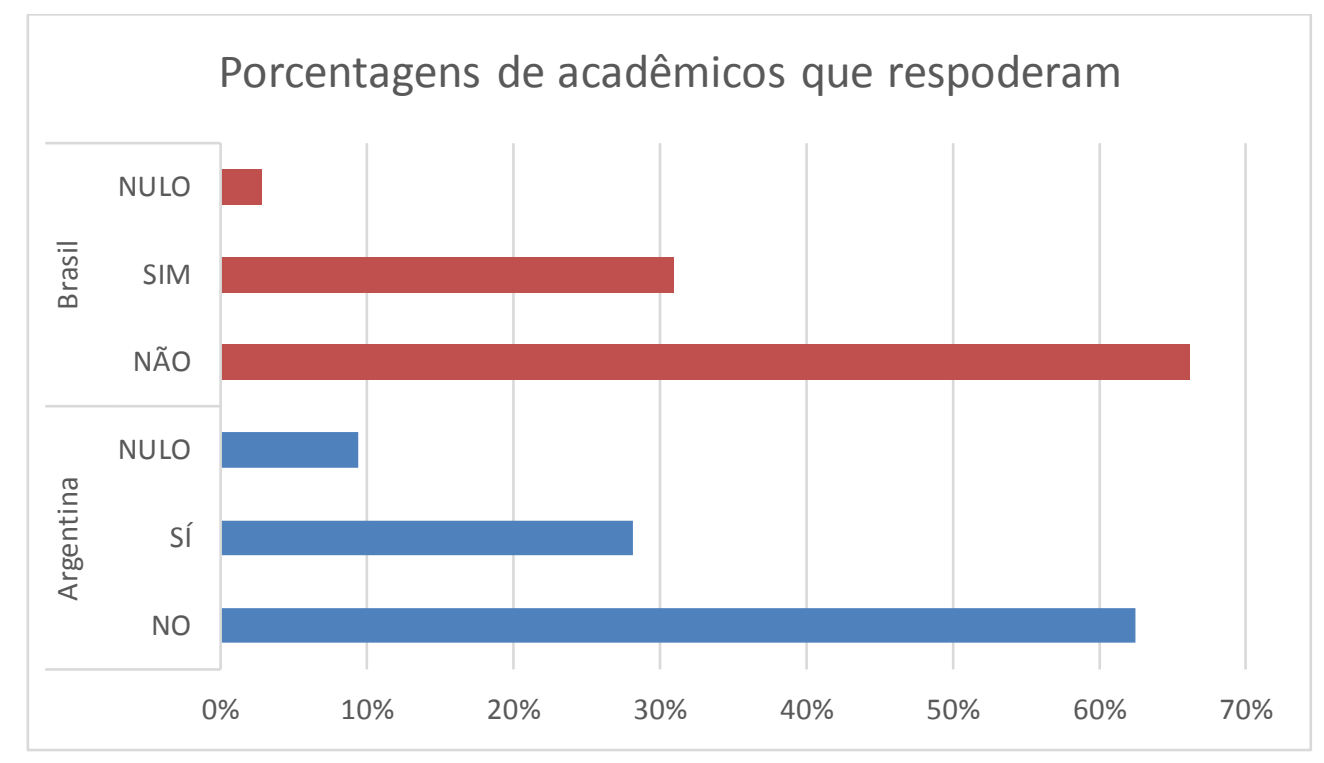

Figura 2: Respostas dos acadêmicos

Uma forma de facilitar o acesso as Coleções Biológicas é através das Coleções virtuais, $3 \%$ dos acadêmicos brasileiros responderam que não conheciam, $31 \%$ que sim e $66 \%$ responderam que não conheciam, em relação aos acadêmicos argentinos foi obtido uma porcentagem de $9 \%$ que responderam que não conheciam, $28 \%$ que conheciam e $63 \%$ alegaram não conhecer Coleções Biológicas virtuais.

As coleções constituem uma fonte de informação para todos os que, por sua atividade, têm contato com seres vivos. Isto envolve áreas estratégicas de atuação governamental, como a gestão do meio ambiente, a pesquisa agronômica, médica ou farmacêutica que possui impacto em toda a sociedade. (ZAHER et. Al). Em relação as Coleções Biológicas Peixoto e et Al afirma que:

O advento das novas tecnologias de informática tem permitido o compartilhamento de dados digitalizados de espécimes biológicos depositados em acervos científicos internacionais, pois a prática do estudo da biodiversidade tropical era preponderantemente realizada por meio de expedições científicas realizadas por instituições científicas de países com tradição mais antiga em pesquisa (PEIXOTO et. $\mathrm{Al})$

Questão 2.1.1 - Já fez uso das coleções biológicas virtuais? 


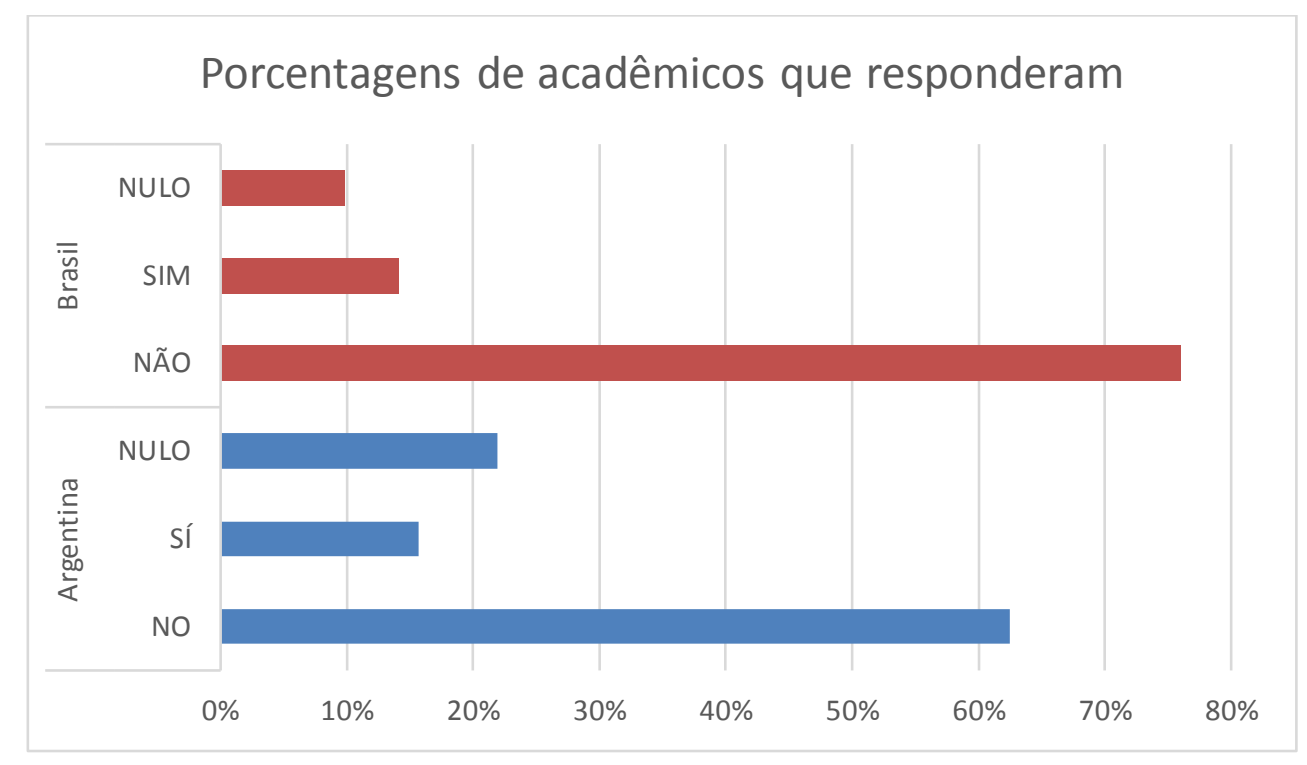

Figura 3: Respostas dos acadêmicos

Foi questionado aos acadêmicos se haviam utilizados coleções biológicas foi obtido como resposta que $10 \%$ dos acadêmicos brasileiros responderam nulo, 14\% que sim e $63 \%$ que não, enquanto os acadêmicos argentinos $22 \%$ optaram pelo nulo, $16 \%$ respondeu que já havia utilizado e $63 \%$ que não.

Através das coleções biológicas é possível tornar o ensino de disciplina de Ciência mais significativos e atrativos para os alunos, propiciando um contato direto com o material, realizando também o manuseio através da coleta dos espécimes. (WOMMER, 2013)

Questão 2.2.1 Se marcou que sim, quais usou e para quê?

$87 \%$ dos acadêmicos brasileiros responderam nulo, $13 \%$ responderam que usaram para atividades, $13 \%$ para TCC/Fungos identificação, 13\% com materiais de oficina, 13|\% com caixa entomológicas, 13\% Coleções Biológicas para divulgação, 13\% Flora do Brasil, identificação, $13 \%$ insetos para divulgação de resultados, $13 \%$ não foi possível identificar a resposta. $75 \%$ dos acadêmicos argentinos deixaram a questão nula, $11 \%$ para estudar, $11 \%$ decalques de crânios, $11 \%$ plantas, $11 \%$ reinos de seres vivos, $11 \%$ anatomia comprada e $11 \%$ responderam que utilizaram em zoologia geral. 
Questão 2.2.2 Em qual situação as utilizou?

Quando questionados em qual situação haviam utilizado as Coleções Biológicas 87\% dos acadêmicos brasileiros deixaram a questão nula, $11 \%$ responderam que utilizaram em oficina, $11 \%$ em TCC, $11 \%$ em pesquisa de trabalho, $11 \%$ para estudos acadêmicos, $11 \%$ no ensino médio, $11 \%$ em monitoria e estagio voluntario, $11 \%$ em minicurso e $11 \%$ no ensino fundamental. Enquanto os acadêmicos argentinos, 79\% deixam a questão nula, 14\% prova, 14\% estudos, $14 \%$ questão para estudos, $14 \%$ estudos antes da prova, 14\% biologia, $14 \%$ botânica e $14 \%$ antropologia.

Questão 2.3 Se marcou não, gostaria de poder fazer uso das coleções biológicas virtuais?

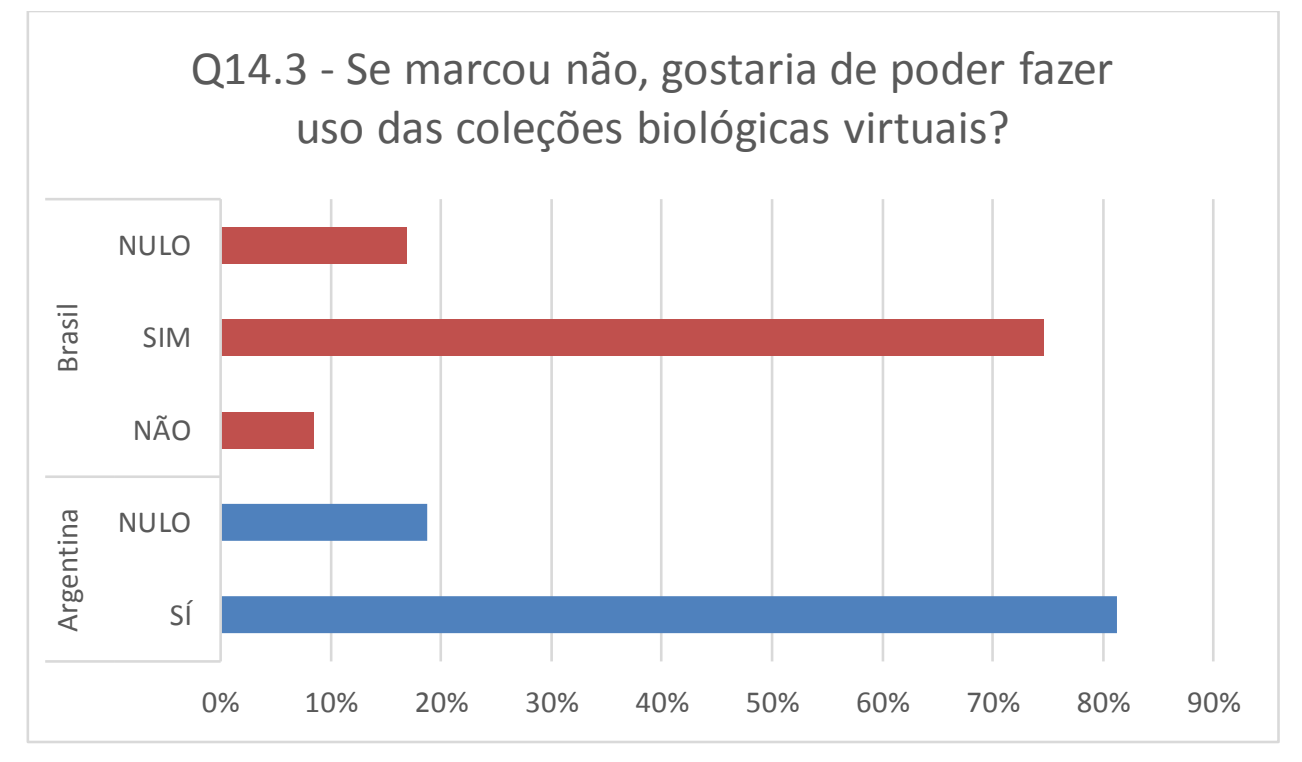

Os acadêmicos que não tiveram oportunidade de utilizar coleções biológicas, foram questionados se havia interesse de utilizar, 17\% responderam nulo, 75\% dos acadêmicos brasileiros responderam que gostariam fazer uso, $8 \%$ disseram que não possuíam interesse, enquanto os acadêmicos argentinos, $19 \%$ responderam nulo e $81 \%$ responderam que sim.

Analisando todas as questões de modo geral, pode se constatar que ambas as universidades possuem uma defasagem no conhecimento sobre Coleções Biológicas, nas perguntas onde foram questionados se os acadêmicos tinham conhecimento sobre o que era Coleção Biológica, houve uma porcentagem alta de respostas nulas. É possível levantar algumas hipóteses a respeito

A primeira hipótese seria a possível falha durante o ensino, talvez o conteúdo não tenha sido aplicado durante as disciplinas da grade curricular. As atividades experimentais constituem 
uma relevante ferramenta que permite ao professor constatar e problematizar o conhecimento prévio dos seus alunos, estimular a pesquisa, a investigação e a busca da solução de problemas. (PERUZZI 2013)

A segunda hipótese seria a má interpretação dos acadêmicos durante a aplicação dos questionários, tendo como base um número alto de porcentagem de acadêmicos que anularam as questões sobre conhecimentos, entretanto responderam que utilizaram em certas disciplinas durante a grade curricular.

\section{Considerações finais}

De acordo com o artigo $1^{\circ}$ da Lei no 9.795/99 A educação ambiental se refere "aos processos por meio dos quais os processos por meio dos quais o indivíduo e a coletividade constroem valores sociais, conhecimentos, habilidades, atitudes e competências voltadas para a conservação do meio ambiente, bem de uso comum do povo, essencial à sadia qualidade de vida e sua sustentabilidade',

É necessário compreender que a Educação Ambiental está inserida em diferentes contextos que podem ser trabalhados em sala de aula, as coleções biológicas são uma maneira eficiente de atingir o objetivo de despertar o interesse nos alunos em descobrir novos conhecimentos a partir da prática. (WOMMER, 2013)

\footnotetext{
As coleções representam também uma herança cultural; um testemunho da rica história do descobrimento e da expansão da sociedade brasileira em seu território nacional. É nas coleções científicas que encontramos representantes da fauna já extinta, que habitou um dia os ecossistemas alterados de forma irreversível pela ação antrópica. Neste sentido, as coleções constituem uma base de dados essencial para os estudos de caracterização e impacto ambiental. (ZAHER et. Al,)
}

Tendo em vista o exposto, não se deve deixar de considerar que os resultados alcançados tenham sido apenas uma falta de interpretação dos discentes durante o preenchimento dos questionários, mas é necessário questionar se a falta de conhecimento foi movida pelo desinteresse dos acadêmicos ou do próprio corpo docente, tendo em mente a importância das Coleções Biológicas para com a Educação Ambiental, fornecendo um acesso a informações a respeito dos seres vivos. 


\section{Referências}

ARANDA, Arion Tulio. Coleções Biológicas: Conceitos básicos, curadoria e gestão, interface com a biodiversidade e saúde pública. 2014.

GARCIA, Yasmin Mayara de Oliveira, et al. Importância das Coleções Biológicas: Coleção de Referência de Vertebrados da Embrapa Pantanal.

PEIXOTO, Ariane Luana et al. Diretrizes e estratégias para a modernização de coleções biológicas brasileiras e a consolidação de sistemas integrados de informação sobre biodiversidade.

PERUZZI, Sarah Luchesse. A importância da aula prática para a construção significativa do conhecimento: a visão dos professores das ciências da natureza. Dispinível em: < http://www.revistaea.org/pf.php?idartigo=1754>. Acesso em 28 dez. 2017

WOMMER, Fernanda Gabriela Bitencourt. Coleções biológicas como estratégia para a educação biológica. Rio Grande do Sul, 2013.

YOUNG, Paulo; ZAHER, Hussam. As coleções zoológicas Brasileiras: Panorama e desafio. 